\title{
Competing forces in liquid metal electrodes and batteries
}

\author{
Rakan F. Ashour ${ }^{\mathrm{a}}$, Douglas H. Kelley ${ }^{\mathrm{a}}$, Alejandro Salas ${ }^{\mathrm{b}, \mathrm{c}}$, Marco Starace $^{\mathrm{b}}$, \\ Norbert Weber ${ }^{\mathrm{b}}$, Tom Weier ${ }^{\mathrm{b}}$ \\ ${ }^{a}$ University of Rochester, 235 Hopeman Building, Rochester, NY 14627, USA \\ ${ }^{b}$ Helmholtz-Zentrum Dresden - Rossendorf, Bautzner Landstr. 400, Dresden, Germany \\ ${ }^{c}$ Instituto Tecnológico y de Estudios Superiores de Monterrey, Monterrey, Mexico
}

\begin{abstract}
Liquid metal batteries are proposed for low-cost grid scale energy storage. During their operation, solid intermetallic phases often form in the cathode and are known to limit the capacity of the cell. Fluid flow in the liquid electrodes can enhance mass transfer and reduce the formation of localized intermetallics, and fluid flow can be promoted by careful choice of the locations and topology of a battery's electrical connections. In this context we study four phenomena that drive flow: Rayleigh-Bénard convection, internally heated convection, electro-vortex flow, and swirl flow, in both experiment and simulation. In experiments, we use ultrasound Doppler velocimetry (UDV) to measure the flow in a eutectic $\mathrm{PbBi}$ electrode at $160{ }^{\circ} \mathrm{C}$ and subject to all four phenomena. In numerical simulations, we isolate the phenomena and simulate each separately using OpenFOAM. Comparing simulated velocities to experiments via a UDV beam model, we find that all four phenomena can enhance mass transfer in LMBs. We explain the flow direction, describe how the phenomena interact, and propose dimensionless numbers for estimating their mutual relevance. A brief discussion of electrical connections summarizes the engineering implications of our work.
\end{abstract}

Keywords: liquid metal battery, electro-vortex flow, internally heated convection, swirl flow, Rayleigh-Bénard convection 


\section{Introduction}

Electrical grids work by balancing the level of demand and supply. Today, the fluctuations are typically handled by gas-fired turbines, which are turned on during the peak demand of electricity. The electrical grid must be designed for that peak power since it lacks an efficient storage mechanism to dampen the load. The increasing integration of renewable energy sources will likely cause even higher fluctuations that cannot be handled by the grid in its current state [1, 2]. Large scale stationary energy storage would likely become mandatory to compensate the fluctuations. Due to its low price, the liquid metal battery (LMB) might be an ideal storage technology [3, 4].

An LMB is composed of two liquid metals with different electronegativity separated by a layer of molten salt electrolyte (Fig. 11a). Typical chemistries include Ca||Bi [5, 6], Ca||Sb [7, 8], K||Hg [9], Li || $\mathrm{Bi}$ [10, 11], Li || $\mathrm{Cd}$ [12], Li||Pb [4, 12, Li||Sb 44, Li||Se [13, 14, Li||Sn [15, 16], Li||Te [17], Li||Zn [12], Mg||Sb [18], Na||Bi [19, 20], Na||Hg [21, 22], Na||Pb [12], Na||Sb [3], Na||Sn [9, 23, 24], $\mathrm{Na}|| \mathrm{Zn}[25,26]$ and $\mathrm{Zn} \| \mathrm{Bi}, \mathrm{Sn}, \mathrm{Pb}$ [27, 28] cells. The three liquid layers are selfsegregated based on density, which makes the manufacturing process simpler and less expensive compared to other batteries. We will consider LMBs of cylindrical shape, though rectangular shapes are also possible. When the battery is connected to an external load, alkali or alkaline metal ions $\left(\mathrm{A}^{+}\right)$are produced via oxidation. These metal ions have to travel through the molten electrolyte before they are reduced at the surface of the positive electrode (B) where they form an alloy $A_{\text {in } B}$. The open circuit potential of the battery depends on the Gibbs free energy of the alloying reaction [3]. The all-liquid design allows for fast charge transfer kinetics [29] and current densities up to $13 \mathrm{~A} \mathrm{~cm}^{-2}$ [12 14. At low currents, the ohmic losses in the electrolyte layer are the most important overpotential. However, at higher current densities concentration polarization becomes the main challenge [9, 21, 24, 30]; due to the slow diffusion of reaction products [3, 18], intermetallic phases are formed at the cathode-electrolyte interface [12, 31]. Often they float on top of the

cathode metal because of their low density [8], and sometimes dendrites may even short-circuit the cell [5]. The solid intermetallic layer will not only hamper mass transfer, but will also act as a solid wall slowing down the flow in the cell. A local accumulation of intermetallic phases will lead to a modified conductivity distribution, causing small scale electro-vortex flow. Hence, a gentle mixing of the bottom electrode may crucially increase cell performance. 


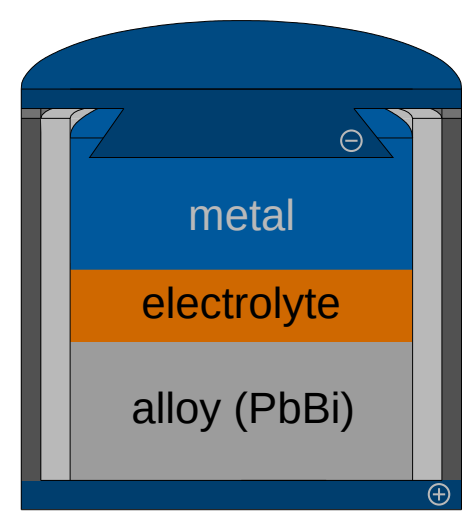

(a)

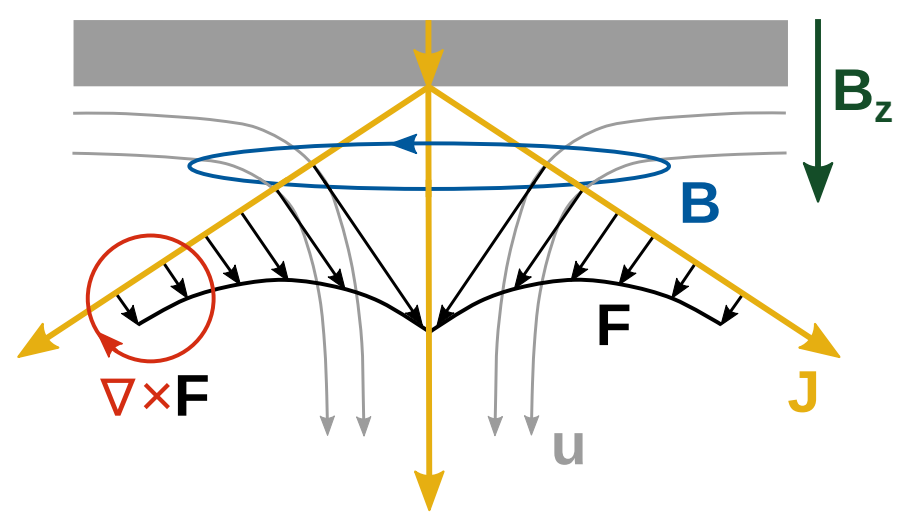

(b)

Figure 1: Sketch of a typical liquid metal battery, which is cylindrical and shown in cross-section (a), and electro-vortex flow at a point current source (b). Diverging current produces a force with nonzero curl that necessarily drives flow, and current always diverges from the negative current collector of a liquid metal battery.

Fluid flow in individual cells does not only affect the cell's efficiency. Perhaps more importantly, the development of fast flows imposes a risk of interface deformations that can lead to an electrical short and sudden discharge of the cell. Prior work in that context focused on the Tayler instability (TI) [32 39], which will appear first in the top electrode. The TI may be suppressed by different means [32, 35] and is likely to short-circuit only very large cells (with a diameter on the order of meters) [36]. In contrast, magnetohydrodynamic (MHD) instabilities of the metal-electrolyte interfaces may appear in small cells, too. Well known from aluminum reduction cells, this "sloshing" or "metal pad roll" instability can be explained by the interaction of horizontal currents with a magnetic background field [40-43]. Small density differences between metal and electrolyte and strong vertical magnetic fields promote this instability [43, 44. In LMBs, the two liquid interfaces can influence each other and may lead to very complex coupling [45, 46] which is still under investigation. Further, thermal convection (TC) is expected to appear especially in the electrolyte and upper metal layer. Although TC will be present in very small cells, its (moderate) flow will not endanger the safe operation of small and medium sized LMBs [47. Marangoni convection driven by a surface tension gradient is not expected to influence the flow velocity considerably, but is expected to change the size of the Rayleigh-Bénard cells [48]. Finally, TC was also found to enhance mass transfer in the positive 
electrode [49, 50], e.g. by heating the cell from below. For a discussion of the thermal balance of an LMB, see [51].

Electro-vortex flow (EVF) can also drive flow in LMBs. Its usefulness for mixing LMBs and its implications for the safe operation of LMBs were already discussed in [52, 53] and [54]. The origin of EVF is best explained by the illustrative example of Shercliff [55] (Fig. 11b). An electric current density $J$ passes from a point source into an infinite fluid layer. Within the fluid, the current diverges radially. The resulting Lorentz force $F$ (which is the cross product of the current density with its own magnetic field $B$ ) is non-conservative, i.e. its curl does not vanish. It can therefore not be balanced by a pressure gradient and drives a flow $\boldsymbol{u}$ away from the wall. This phenomenon is commonly denoted as "electrically driven vortical flow" or shorter "electro-vortex flow". The term "vortex" refers to the typical flow structure observed in confined volumes. EVF is caused by internal currents producing a rotational Lorentz force and driving a jet away from the wall [55]. It cannot exist in 2D [55]. Since battery electrodes are typically wider than the wires that supply their currents, and current collectors must be made small enough to prevent shorts with vessel walls, battery currents almost always diverge, at least in some regions of the electrode. The extent to which they diverge and drive electro-vortex flow depends on connection topology.

Already one year before Shercliff, Lundquist studied the same problem. He discovered that the velocity of EVF scales with the so-called EVF parameter $S=\mu_{0} I^{2} /\left(4 \pi^{2} \rho \nu^{2}\right)$ [56, 57] with $\mu_{0}, I, \rho$ and $\nu$ denoting the vacuum permeability, the current, the density, and the kinematic viscosity, respectively (for different definitions, see [58, 59]; 59] might have a typo). At typical currents for industrial applications the velocity scales as $u \sim \sqrt{S}$, i.e. linearly with the current. Only at low currents, a quadratic scaling is observed [57, 59, 60]. It should be further noted that the jet is steady only at very low velocities; typically, it oscillates (see e.g., [61, 62]). At very high velocities, the flow can generally saturate by the induced current or magnetic field (as, e.g., the Tayler instability [38, 39]). However, at typical velocities, the main dissipation happens in the boundary layers [61, 63] (for an experimental study see [64]). The thoroughly studied problem of an infinite fluid layer [56, 58, 65, 71] (overview [57, 72]) is therefore of limited practical relevance because it involves no boundary layers.

Davidson provides a concise, clear, and understandable introduction to electro-vortex flow [61]. For an even shorter introduction, see Chudnovskii 
73 and for a broad overview on EVF and its application Bojarevics et al. [60]. A number of experiments on electro-vortex were conducted by Woods et al. [74], Butsenieks et al. [59, 75-77], Bojarevics et al. [78], Zhilin et al. [42, 64, 79] and Räbiger et al. [80 82]; for an excellent overview, see [60].

At low currents the jet illustrated in Fig. 1p usually forms a poloidal flow that descends along the axis of the cylindrical electrode and rises along the walls, since it is confined by boundaries. However, at high currents an azimuthal flow or "spontaneous swirl" 61] is often observed experimentally [74, 83. Its origin can be explain similar to the Sele mechanism in aluminum reduction cells [40, 43]. A vertical magnetic background field and the radial cell current produce an azimuthal force driving an azimuthal flow. The magnetic background field may be the Earth's magnetic field [83]; similarly a "slight deviation from symmetry" in the experiment will lead to swirl [62, 63, 78]. Making electrical connections among a tightly-packed array of batteries is almost certain to cause such deviations from symmetry. The spin-up of swirl takes a significant time (around a minute in large vacuum arc remelting vessels) [84]; therefore, swirling may be minimized by periodically reversing the direction of the vertical background field [84].

Curiously, the swirl is known to almost totally supersede the poloidal flow. Davidson explains that "the radial stratification of angular momentum suppresses radial motion in the same way as density stratification suppresses axial motion" 84]. He demonstrates that the azimuthal flow suppresses the poloidal one ("poloidal suppression") and argues that Ekman pumping [85] is the key dissipative mechanism [63]. He further predicts that an azimuthal force of only $1 \%$ of the poloidal one will lead to a strong swirl flow. Alternative explanations have also been proposed, however [76, 83, 86].

In this article we study the flow in the bottom electrode only. Specifically we investigate the interaction of four different flow phenomena: electro-vortex flow, swirl flow, internally heated convection (IHC) and classical RayleighBénard convection. All four depend strongly on the location and topology of electrical connections to the battery. Those connections are likely to be tightly packed into a large array of batteries, since the technology is intended to store enough energy for neighborhoods or cities. Where the connections enter and leave each battery, how much they concentrate the electrical current, and what paths they take outside the batteries can all affect flow in the electrode. To study the effects of connection on the four flow phenomena, we use a combination of experimental and numerical models of $\mathrm{PbBi}$ electrodes operating at mean current densities of up to $0.65 \mathrm{~A} \mathrm{~cm}^{-2}$. 
The paper proceeds as follows: in the next section we describe the experimental apparatus and numerical model, followed by an order of magnitude estimation of the different flows in section 3. Finally we present and discuss the results in section 4 .

\section{Methods}

\subsection{Experimental}

A schematic of the experimental apparatus is shown in Fig. 2, The vessel is a cylinder of 304 stainless steel with inner radius $R=44.5 \mathrm{~mm}$ that also serves as the positive current collector. The liquid metal used in the experiments is a eutectic alloy of $44.5 \%$ lead and $55.5 \%$ bismuth. The vessel rests on an aluminum plate which is much more conductive than stainless steel, thereby allowing heat and current density to homogenize before entering the vessel. The upper current collector is a $4 \mathrm{~mm}$-diameter nickel-plated copper wire with mica and fiberglass insulation. It is mounted on a PTFE sheet using shaft collars and is connected to a current-controlled DC power source. Two K-type thermocouples are placed at different depths to measure the temperature difference between the top and bottom of the liquid metal electrode. A third thermocouple is connected to a proportional-integral-derivative controller, which allows for the temperature to be maintained at $160{ }^{\circ} \mathrm{C}$ (at the bottom of the cell). The temperature is chosen to match the operating conditions of a $\mathrm{Na} \| \mathrm{PbBi}$ battery [87]. The apparatus minimizes heat exchange by using ceramic insulation, which allows for the operating temperature to be maintained for the duration of the experiment [see 49, 88].

Since liquid metal is opaque, it is not possible to measure flow using optical methods like particle tracking or particle image velocimetry. Therefore, to study mixing and transport in liquid metal electrodes we use an ultrasound Doppler velocimeter (Signal-Processing DOP 3010). The velocimeter drives an ultrasound probe that makes a beep and listens for echoes. The distance of an echoing body is determined from the speed of sound and the time delay between emitted and received signals. The speed of that body toward or away from the probe is determined from the Doppler shift of the echo. Negative values in the velocity profile indicate a flow moving toward the probe, whereas positive values indicate a flow away from the probe. Small oxide particles or other impurities common in liquid metal provide sufficient echoes. The probe operates with a frequency of $8 \mathrm{MHz}$ and has a piezoelectric 

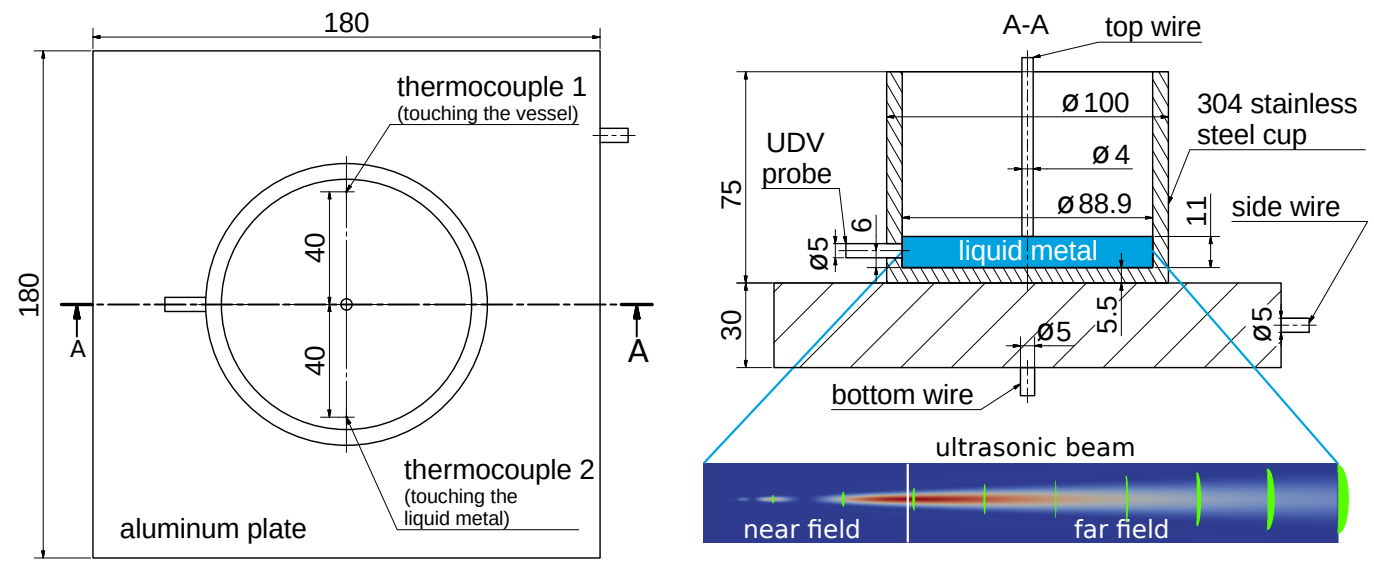

Figure 2: Dimensions of the experiment and simulation model (in $\mathrm{mm}$ ) and illustration of the ultrasound beam showing the near and far field as well as the beam broadening.

emitter of diameter $5 \mathrm{~mm}$, which gives a radial resolution of $0.5 \mathrm{~mm}$ and lateral resolution of a few $\mathrm{mm}$. In the apparatus shown in Fig. 2, the ultrasound probe is mounted on the side of the cylinder allowing for the measurement of the radial velocity component of the flow along a diameter, $6 \mathrm{~mm}$ above the vessel floor. The cylinder is filled with $700 \mathrm{~g}$ of eutectic $\mathrm{PbBi}$, which gives a realistic electrode thickness of about $11 \mathrm{~mm}$. Though UDV measurements in similar shallow layers were already successfully conducted in the past [89], our experiment is fairly challenging. As the UDV beam broadens on its way (Fig. 2), it samples flow in a finite volume in which the velocity is not necessarily uniform; regions of flow towards and away from the probe may cancel each other out. In order to correctly compare the simulated velocities, we use a simple UDV beam model (Appendix A).

To study the effect of electric current topology on fluid flow, two different current paths are investigated. In one arrangement, current is supplied to the side of the aluminum plate. This model is very likely to be seen in battery packs (see e.g., Fig. 20 in [52, 53]). In the other arrangement, current is supplied to the bottom of the aluminum plate through a $100 \mathrm{~cm}$ long aluminum rod. This arrangement represents vertically stacked cells and minimizes symmetry-breaking in the supply currents, thereby reducing swirl flow. 


\subsection{Mathematical and numerical model}

In our simulations, thermal convection and electro-vortex flow are modeled separately. The two models are implemented in the open source library OpenFOAM [90]. If not otherwise stated, the following material properties are assumed for eutectic $\mathrm{PbBi}$ at $160^{\circ} \mathrm{C}$ : a kinematic viscosity of $\nu=2.7 \cdot 10^{-7} \mathrm{~m}^{2} \mathrm{~s}^{-1}$, a volumetric thermal expansion coefficient of $\beta=$ $1.3 \cdot 10^{-4} \mathrm{~K}^{-1}$, an electric conductivity of $\sigma=9 \cdot 10^{5} \mathrm{~S} \mathrm{~m}^{-1}$, a density of $\rho=10505 \mathrm{~kg} \mathrm{~m}^{-3}$, an isobaric heat capacity of $c_{p}=148 \mathrm{~J} \mathrm{~kg}^{-1} \mathrm{~K}^{-1}$, a heat conductivity of $\lambda=10 \mathrm{~W} \mathrm{~m}^{-1} \mathrm{~K}^{-1}$, a thermal diffusivity $\alpha=6 \cdot 10^{-6} \mathrm{~m}^{2} \mathrm{~s}^{-1}$, a Prandtl number of $\operatorname{Pr}=0.04$ and a sound velocity of $c=1765 \mathrm{~m} \mathrm{~s}^{-1}$ [91 93 . The electric conductivity of the vessel is assumed to be $\sigma=1.37 \cdot 10^{6} \mathrm{~S} \mathrm{~m}^{-1}$ and that of the aluminum plate $\sigma=3.5 \cdot 10^{7} \mathrm{~S} \mathrm{~m}^{-1}$.

\subsubsection{Thermal convection}

Thermal effects are modeled in the fluid only. All solid conductors are replaced by simplified boundary conditions. The flow is simulated using the Oberbeck-Boussinesq approximation [94]. Its validity is estimated according to Gray and Giorgini [95]: a temperature difference of $27 \mathrm{~K}$ would lead to an error of $10 \%$ of the dynamic viscosity, when neglecting its temperature dependence. Respectively, the temperature difference may reach $66 \mathrm{~K}$ for thermal conductivity and $833 \mathrm{~K}$ for $c_{p}$ and density in order to stay below the same error. As we expect temperature differences in the order of $10 \mathrm{~K}$, the Oberbeck approximation is well-suited for our problem. The Navier-Stokes and energy equations

$$
\begin{aligned}
\frac{\partial \boldsymbol{u}}{\partial t}+\nabla \cdot(\boldsymbol{u u}) & =-\nabla p_{d}+\nu \Delta \boldsymbol{u}-\boldsymbol{g} \cdot \boldsymbol{r} \nabla \rho_{k} \\
\nabla \cdot \boldsymbol{u} & =0 \\
\frac{\partial T}{\partial t}+\nabla \cdot(\boldsymbol{u} T) & =\frac{\lambda}{\rho_{0} c_{p}} \Delta T+\frac{\boldsymbol{J}^{2}}{\sigma \rho_{0} c_{p}}
\end{aligned}
$$

are solved, with $\boldsymbol{u}, t, \boldsymbol{g}, \boldsymbol{r}, T$, and $\boldsymbol{J}$ denoting velocity, time, gravitational acceleration, position, temperature, and current density, respectively. The density $\rho=\rho_{0} \rho_{k}=\rho_{0}\left(1-\beta\left(T-T_{\text {ref }}\right)\right)$ is calculated using the mean density $\rho_{0}$ at reference temperature $T_{\text {ref }}$ and the coefficient of thermal expansion $\beta$. The current density is computed with the electro-vortex solver and provided as initial condition. The modified pressure is defined as $p_{d}=(p-\rho \boldsymbol{g} \cdot \boldsymbol{r}) / \rho_{0}$, where $p$ is the pressure. The set of equations is solved using the PISO algorithm on a collocated grid (with Rhie-Chow interpolation [96]) employing a 
modified version of the OpenFOAM standard solver buoyantBoussinesqPimpleFoam and using at least 300 cells on the diameter.

\subsubsection{Electro-vortex flow}

Simulating electro-vortex flow requires the coupling of solid and liquid conductors to obtain the correct current density. This coupling is achieved by a parent-child mesh technique. While electric properties (electric potential, current density) are solved on a global mesh, the flow is computed in the fluid region only, so quantities must be mapped between meshes. The magnetic field is determined by an integro-differential approach, i.e. its boundary conditions are computed using Biot-Savart's integral [34] and the induction equation is solved in the fluid. The model is described in detail in [97].

The electric potential $\Phi$, current density $\boldsymbol{J}$ and magnetic field $\boldsymbol{B}$ are split into constant (subscript 0) and induced parts (lower case) as

$$
\begin{aligned}
\Phi & =\Phi_{0}+\varphi \\
\boldsymbol{J} & =\boldsymbol{J}_{0}+\boldsymbol{j} \\
\boldsymbol{B} & =\boldsymbol{B}_{0}+\boldsymbol{b} .
\end{aligned}
$$

The induced magnetic field $\boldsymbol{b}$ is neglected. The following set of equations is solved on the global mesh:

$$
\begin{aligned}
\nabla \cdot \sigma \nabla \Phi_{0} & =0 \\
\nabla \cdot \sigma \nabla \varphi & =\nabla \cdot \sigma(\boldsymbol{u} \times \boldsymbol{B}) \\
\boldsymbol{J}_{0} & =-\sigma \nabla \Phi_{0} \\
\boldsymbol{j} & =\sigma(-\nabla \varphi+\boldsymbol{u} \times \boldsymbol{B}) .
\end{aligned}
$$

In the fluid region we solve

$$
\begin{aligned}
& \frac{\partial \boldsymbol{u}}{\partial t}+(\boldsymbol{u} \cdot \nabla) \boldsymbol{u}=-\nabla p+\nu \Delta \boldsymbol{u}+\frac{\boldsymbol{J} \times \boldsymbol{B}}{\rho} \\
& \boldsymbol{B}_{0}(\boldsymbol{r})=\frac{\mu_{0}}{4 \pi} \int \frac{\boldsymbol{J}_{0}\left(\boldsymbol{r}^{\prime}\right) \times\left(\boldsymbol{r}-\boldsymbol{r}^{\prime}\right)}{\left|\boldsymbol{r}-\boldsymbol{r}^{\prime}\right|^{3}} d V^{\prime} \\
& 0=\Delta \boldsymbol{B}_{0}
\end{aligned}
$$

with $\mu_{0}$ and $V$ denoting the vacuum permeability and cell volume, respectively. Computing Biot-Savart's integral, the current $\boldsymbol{J}$ of the full mesh is used for determining $\boldsymbol{B}$ at the fluid boundaries only. The grid resolution is approximately 100 cells on the diameter with strongly refined boundary layers. 


\section{Theory of transport in liquid metal electrodes}

In this section, we will derive two simple dimensionless numbers for estimating which flow dominates: thermal convection or electro-vortex flow. We will start with classical Rayleigh-Bénard convection.

A typical liquid metal battery setup requires heating a cylindrical container from the bottom, which generates a temperature gradient along the depth of the electrode. As the liquid closer to the bottom becomes less dense, it rises to the surface allowing for the cooler fluid to sink down. A steady flow is established when the balance of kinetic energy and viscous dissipation is reached. In systems where the viscosity is high enough, or length scales are small enough, fluid motion can be completely suppressed. The onset of thermal convection is defined by the Rayleigh number (i.e. the ratio of buoyant to viscous forces) as

$$
R a=\frac{g \beta h^{3}\left(T-T_{0}\right)}{\alpha \nu}
$$

with $g, h$, and $T-T_{0}$ denoting the gravitational acceleration, the height of the layer, and the temperature difference, respectively, all at working temperature $T_{0}=160{ }^{\circ} \mathrm{C}$. In our specific experiment with a free upper surface, convection is expected to set in at a critical Rayleigh number of $R a_{\text {cr }}=1100$ [98], which corresponds to a temperature difference of $\Delta T \approx 1 \mathrm{~K}$. We expect therefore strong Rayleigh-Bénard convection in the experiment, which we maintain at a temperature difference of $\Delta T \approx 8 \mathrm{~K}$. The typical velocity scale of Rayleigh-Bénard convection can be estimated by the free fall velocity as [47, 99]

$$
U_{b} \sim \sqrt{g \beta \Delta T h}
$$

We will now proceed with the case of internally heated convection (IHC), which is caused by Joule heating of the electric current due to ohmic losses. Similarly, the free fall velocity may be used as velocity scale. However, some characteristic temperature difference needs to be defined first. Following

Goluskin [47, 100] we can use the heating rate $\dot{Q}^{\prime}=J^{2} /\left(\sigma \rho c_{p}\right)$ [101] to obtain a characteristic temperature gradient

$$
\Delta T=\frac{h^{2} J^{2}}{\alpha \sigma \rho c_{p}} .
$$


Finally, we consider electro-vortex flow. When an electrical current density $\boldsymbol{J}$ is injected into the fluid, it induces a magnetic field $\boldsymbol{B}$, and the two are related via Ampere's law as

$$
\oint_{C} \boldsymbol{B} d \ell=\mu_{0} \int_{S} \boldsymbol{J} d S,
$$

with $S, C$ and $d l$ denoting a surface, the curve around the surface and an infinitesimal element of the curve. In geometries relevant for liquid metal batteries, electrical current flows between two current collectors, both aligned on the axis of symmetry. Typically, the upper current collector has a smaller diameter than the lower one. Given that geometry, we first consider purely axial current, which would drive a circulation that rises near the side walls and descends near the center. To see why, apply $(17)$ to two paths of integration, both circles of the same radius and both aligned with the central axis, but at different heights. Since current is more concentrated near the smaller, upper current collector, the magnetic field there is larger. The Lorentz force points toward the central axis everywhere; however, it has larger magnitude near the upper current collector, causing an inward flow there. Conservation of mass then requires outward flow near the lower current collector, sinking near the center, and rising near the side walls. Arguments made with this poloidal circulation in mind lead to an expected scaling of electro-vortex flow as 61]

$$
U_{L} \sim \frac{\left(\mu_{0} / \rho\right)^{1 / 2} I}{2 \pi R},
$$

with $\mu_{0}, I$ and $R$ denoting the vacuum permeability, the cell current and radius, respectively. For other (but similar) possible scales, see [59, 60, 77, 102 .

In a flow in which both buoyant and electromagnetic forces are present, we expect flow speed to be predicted by (15) when buoyancy dominates and by (18) when electromagnetic forces dominate. We can moreover estimate which of the two effects dominates. The ratio of the characteristic velocities of electro-vortex flow to Rayleigh-Bénard convection is

$$
A \sim \frac{\left(\mu_{0} / \rho\right)^{1 / 2} I}{2 \pi R \sqrt{g \beta \Delta T h}}
$$

and the ratio of the characteristic velocities of electro-vortex flow to internally 
heated convection

$$
B \sim \frac{R}{2 h} \sqrt{\frac{\mu_{o} \sigma \lambda}{\rho g \beta h}} .
$$

For alternative expressions, see [101, 103, 104. We can think of equation (19) as the ratio of momentum gained from electromagnetic forces to that gained from buoyant forces in the core of the electrode, whereas equation (20) can be thought of as the ratio of momentum gained by electromagnetic forces to that dissipated by Joule heat. Hence, when $A \ll 1$ or $B \ll 1$, we expect buoyancy to dominate the flow. When $A \gg 1$ and $B \gg 1$, we expect electro-vortex flow to dominate.

The dimensionless ratios $A$ and $B$ are obtained from a simple formalistic approach. Compared to the sophisticated considerations of Davidson et al. for a similar problem [84], our approach is quite simplistic - probably too simple. Most notably, our formulas do not cover the distribution of the current density nor the diameter of the current collectors. These will - of course - have a crucial influence on the flow magnitude, though they will not change $A$ and $B$. Nevertheless, we believe it is admissible to use $A$ and $B$ as a first estimate for the relative importance of thermal convection and electrovortex flow. Table 1 shows both dimensionless quantities for our geometry for eutectic PbBi as well as liquid sodium. Considering the low values of $A$, we expect Rayleigh-Bénard convection to dominate in the experiment. This is indeed the case, as we will show later. On the other hand, the dimensionless quantity $B$ is clearly larger than one. Thus, we expect electro-vortex flow to dominate over internally heated convection. In experiments, however, the unavoidable presence of classical Rayleigh-Bénard convection does not allow comparing EVF and IHC directly.

Finally, a third dimensionless ratio can be used to estimate the transition from poloidal electro-vortex to an azimuthal swirl flow. As its derivation is effectively explained by Davidson et al. [61, 63, we give here only the result: if an azimuthal force of a magnitude of $1 \%$ of the poloidal one is present, we expect swirl flow to dominate.

\section{Results and discussion}

In this section we present the measured and simulated velocities in the liquid electrode without any current (Fig. 3), with $I=2 \mathrm{~A}$ (Fig. 4) and 
Table 1: Dimensionless parameters for different electrode materials using the same geometry as in the experiment $(I=40 \mathrm{~A}, R=44.5 \mathrm{~mm}, h=11 \mathrm{~mm}, \Delta T=8 \mathrm{~K})$. For the material properties, see $91,93,105,106$.

\begin{tabular}{ccc}
\hline Component & $\mathrm{A}$ & $\mathrm{B}$ \\
\hline Na anode & 0.36 & 386 \\
PbBi cathode & 0.15 & 17.5 \\
\hline
\end{tabular}

$I=40$ A (Fig. 5). In all experiments, velocity profiles are recorded when the temperature of the vessel is stabilized at $160{ }^{\circ} \mathrm{C}$. The measured temperature difference between the top and bottom of the liquid metal was always in the range of $7 \mathrm{~K} \leq T-T_{0} \leq 9 \mathrm{~K}$.

Figure 3 a shows the measured radial velocity distribution along the diameter of the electrode recorded for two minutes. No current is applied; we observe only Rayleigh-Bénard convection. The flow is rather disordered. This fits well to the numerical results (Fig. 35), obtained with no-slip boundary conditions for velocity, a fixed vertical temperature gradient and adiabatic side walls. (The oxide film formed on the melt justifies using a no-slip boundary condition for the free surface [107].) Using the ultrasound beam model described in Appendix $\mathrm{A}$ we extract the radial velocity projection along the diameter of the UDV probe so that we can directly compare the mean velocity from experimental measurements with numerical simulations. Figure $3 \mathrm{~b}$ shows the mean velocity from experimental and numerical measurements (averaged over $100 \mathrm{~s}$ ). Both agree fairly well with respect to the flow speed and the number of convection cells (illustrated in Fig. $3 \mathrm{~d}$ ). Though the flow is rather disordered, structure may be imposed via pinning of convection cells or heat transfer through the side walls. The top electrode certainly cools the metal bath in the middle. Such a finger cooling is well known to produce an axisymmetric flow in the Czochalski process of crystal growth [107, 108]. Our configuration might also be compared to a flow driven by a localized (external) heat source [109 113].

When a (small) current of $I=2 \mathrm{~A}$ is applied to the cell, the measured flow becomes immediately much more ordered (compare Fig. 3 and 4 a); the measured flow magnitude does not change. Previous work dealing with liquid metal convection in the presence of magnetic fields suggest that convection rolls will tend to align with magnetic field lines, producing a more ordered flow structure [114, 115]. However, there are two other ways to explain the increase in order much better: electro-vortex flow and internally heated con- 
(a)

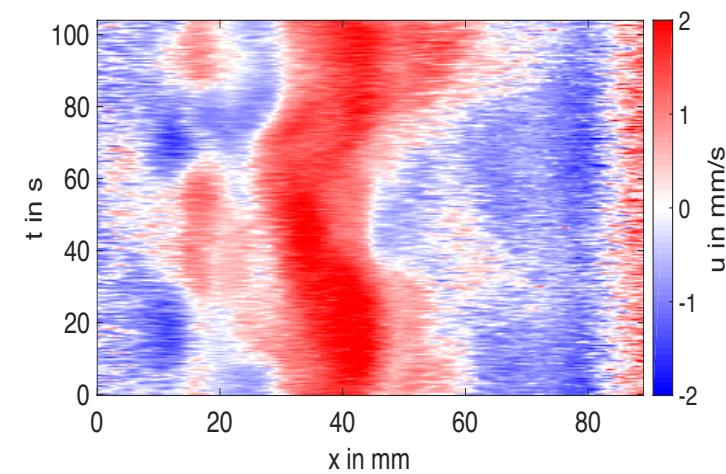

(b)

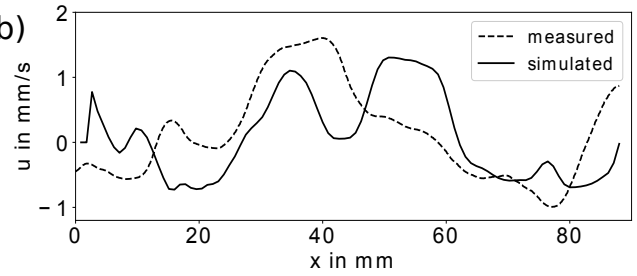

(c)

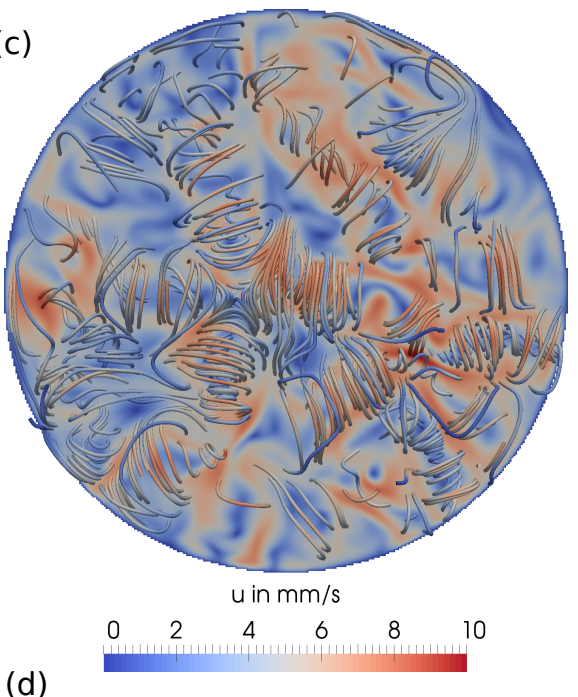

(d)

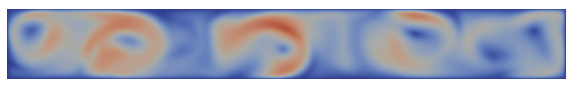

Figure 3: Pure Rayleigh-Bénard convection for $\Delta T=8 \mathrm{~K}$. Measured radial velocity, (a). Mean speed across a diameter of the electrode, $6 \mathrm{~mm}$ above the vessel floor, (b), as measured (dashed curve) and simulated (solid curve). Speed and streamlines on a horizontal cross-section $5.5 \mathrm{~mm}$ above the vessel floor, (c). Speed on the vertical cross-section from which the ultrasound projection is extracted, (d). Rayleigh-Bénard convection drives disordered flow.

vection (IHC). Fig. 4h shows the simulated electro-vortex flow: a downward jet below the upper electrode, exactly as expected. In the same volume, most of the Joule heat will be generated. The strong radial temperature gradient forms lateral jets and several convection cells (Fig. 44). Comparing Figs. 4 $\mathrm{d}$ and e, three things are noteworthy: EVF and thermal convection have an opposing flow direction, the magnitude of the thermal flow is one order of magnitude larger than EVF, and both are fairly difficult to measure in our experiments. In vacuum arc remelting the same flow directions are observed. However, electro-vortex flow reaches - compared to thermal convection a "similar size" [84 of e.g. 30\% of the typical convection speed [101]. A direct comparison of the (time averaged) mean velocity projection along the diameter of the aforementioned flows is shown in Fig. 4 $\mathrm{b}$. The simulated flow profile for IHC matches the measurement well (black curves). However, the simulation shows velocities twice as large as the measured ones. We believe 
(a)

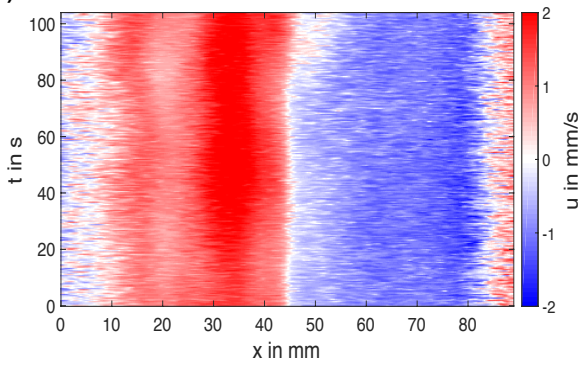

(b)

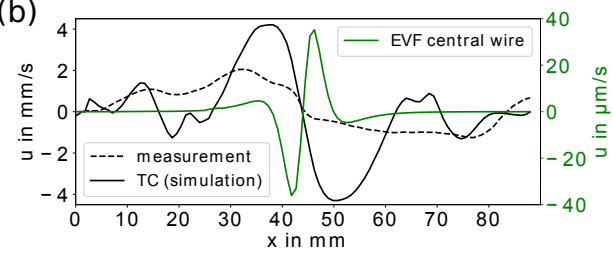

(c)
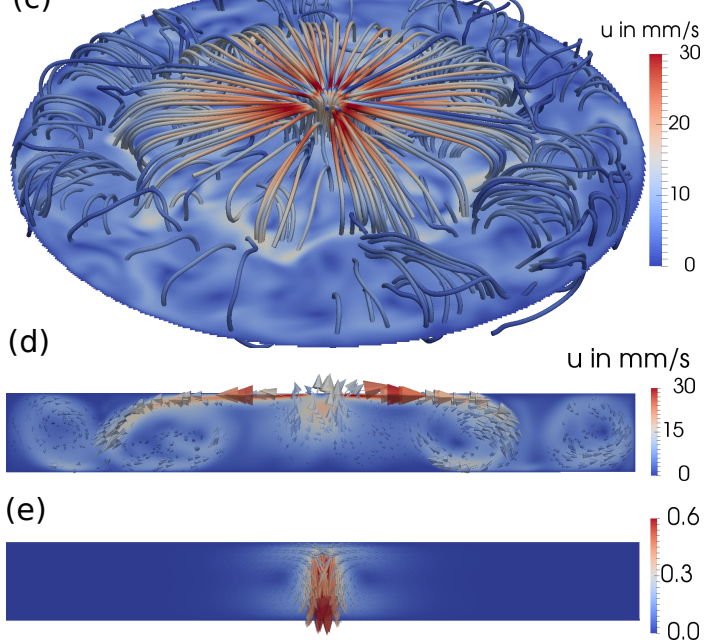

Figure 4: Convection and electro-vortex flow with $I=2 \mathrm{~A}$. Measured radial velocity, (a). Mean speed across a diameter of the electrode, $6 \mathrm{~mm}$ above the vessel floor, (b), as measured (dashed curve) and simulated (solid curves). Speed and streamlines on a horizontal cross-section $5.5 \mathrm{~mm}$ above the vessel floor, (c). Flow due to internally heated convection (IHC) alone, on the vertical cross-section from which the ultrasound projection is extracted, (d). Flow due to electro-vortex flow (EVF) alone, on the same vertical cross-section, (e). IHC dominates EVF and has a shape consistent with experimental observations, but larger magnitude.

this discrepancy may be explained partially by the simplified boundary conditions (no-slip for velocity; fixed vertical temperature difference; adiabatic side-walls). Moreover, an interaction between the opposing EVF and thermal convection may change the flow structure, too. Despite the described challenges of measurement and simulation we can state: internally heated convection dominates the flow.

When $I$ is increased to $40 \mathrm{~A}$, the measured velocities increase by a factor of five (Fig. 5a). We no longer observe a global poloidal circulation, but a tornado-like flow structure emerges - a phenomenon well known from former experiments [60, 74, 83]. Unfortunately, it is not possible to measure the azimuthal flow speed with our current experimental apparatus. Anyway, we know when swirl appears first: at $20 \mathrm{~A}$, if the current is supplied symmetrically and at $10 \mathrm{~A}$ if is supplied from the side. As mentioned in the introduction, the appearance of azimuthal forces can easily be explained: the internal radial currents interact with a vertical stray magnetic field. This 
(a)

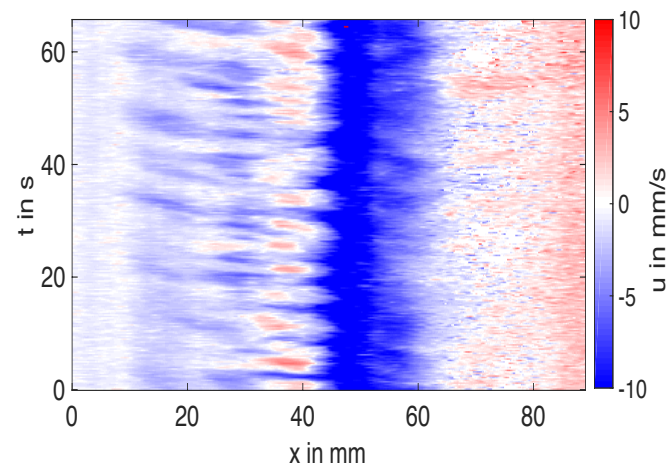

(b)

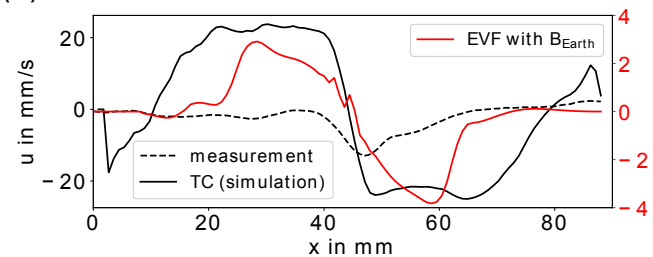

(c)

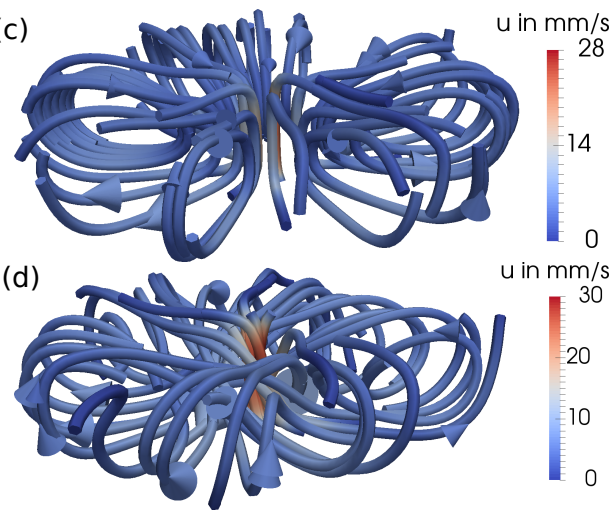

(e)

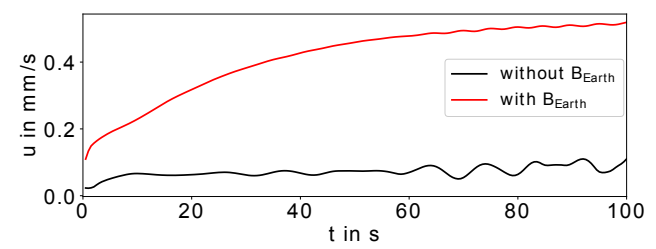

Figure 5: Convection and electro-vortex flow with $I=40$ A. Measured radial velocity, (a). Mean speed across a diameter of the electrode, $6 \mathrm{~mm}$ above the vessel floor, (b), as measured (dashed curve) and simulated (solid curves). Streamlines of electro-vortex flow simulated without Earth's magnetic field (c), and with it (d). Volumetrically-averaged poloidal and azimuthal velocity of electro-vortex flow (e). In (a) and (b), both internally heated convection and Rayleigh-Bénard convection are present. Earth's field causes azimuthal flow in both experiments and simulations, but suppresses poloidal flow more strongly in experiments.

may be the Earth's magnetic field of $B_{z} \approx 0.5 \mathrm{mT}$ or with the words of Bojarevics: the "slightest deviation from symmetry" leads to swirl [78, because it produces such fields [60, 61, 83]. The often observed dominance of the azimuthal flow is harder to explain. While Millere assumes the kinetic energy of the poloidal flow to be "partially transferred to the rotational motion" [76, Shtern finds a supercritical bifurcation of the poloidal flow leading to swirl [86. Davidson et al. point out that the model of the latter author is oversimplified. He explains the dominance of swirl as "poloidal suppression" [63], which is in the sense of [76] and [83]. Specifically, he describes that the swirling motion forces the poloidal flow into a thin, dissipative Ekman layer where it is suppressed efficiently.

In Figs. 55 and d we show the electro-vortex flow, simulated with and without the Earth's magnetic field. The latter we measured in Dresden 


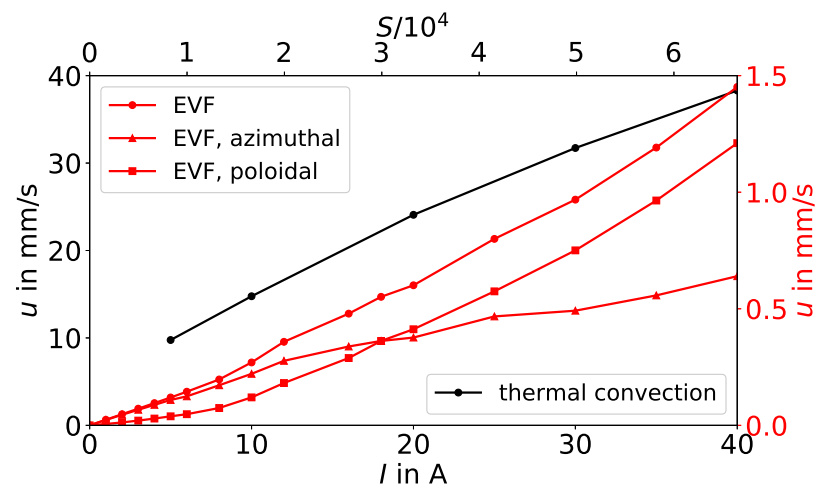

Figure 6: Volume-averaged velocity due to simulated internally heated convection (left axis) and electro-vortex flow (right axis). For these simulations we used symmetric current collectors and $\boldsymbol{B}=\left(15 \cdot \boldsymbol{e}_{x}, 5 \cdot \boldsymbol{e}_{y}, 36 \cdot \boldsymbol{e}_{z}\right) \mu \mathrm{T}$. Though the speeds of both internal thermal convection and electro-vortex flow are predicted to scale linearly with current, we find that both deviate slightly.

as $\boldsymbol{B}=\left(15 \cdot \boldsymbol{e}_{x}, 5 \cdot \boldsymbol{e}_{y}, 36 \cdot \boldsymbol{e}_{z}\right) \mu \mathrm{T}$. Figure 5 illustrates, that indeed the azimuthal velocity increases due to the Earth's magnetic field. In contrast to Davidson, we do not observe a strong poloidal suppression (compare with Fig. 9 in [63]); investigation of that discrepancy is underway. Finally, we compare measurement and simulation in Fig. 5b. Obviously, the profile of the flow matches well, while its magnitude does not. Simulating the effects of thermal convection and EVF separately is important, but it is not enough. Both phenomena interact: presumably the electromagnetically driven swirl flow suppresses the thermal flow as described by Davidson et al. [63. A more detailed experimental and numerical study of that interaction is planned for the near future.

A strong swirl flow may enhance mass transfer, but will also lead to differences in the centripetal pressure along the interface. If strong enough it will deform the electrode-electrolyte interface and may eventually lead to a short circuit of the cell [45, 116]. By this means, swirling electro-vortex flow could trigger interface instabilities in LMBs.

Figure 6 shows the simulated mean flow velocity of internally heated convection (left axis) and electro-vortex flow including the Earth's magnetic field (right axis). As discussed before, thermal convection is one order of magnitude larger than EVF. Though equation (15) and (16) predict that thermal convection speed will increase linearly with current, we find the rate of in- 
crease to be slower. On the other hand, the global EVF speed grows slightly faster than linearly with current. According to Vlasyuk [59, 60] we would expect a quadratic scaling up to $S=1000$ and above $S=10^{5}$ a linear scaling. Operating in the transition region between both, our results seem to fit roughly to Vlasyuks theoretical prediction. Figure 6 also shows the poloidal and azimuthal mean velocity of EVF. The poloidal mean velocity scales approximately quadratically with current, as one might expect. Increasing the cell current, we also simultaneously increase the magnetic field induced by the current itself, with which the current interacts to drive poloidal flow. The rather linear scaling of the azimuthal flow can be explained in a very similar way. Here, only the cell current - together with the constant vertical field of the Earth — drives the flow.

\section{Summary, design implications, and outlook}

Using a combination of in-situ ultrasound velocity measurement and numerical simulations, we studied the flow in a liquid $\mathrm{PbBi}$ electrode. Specifically, we explored classical Rayleigh-Bénard convection, internally heated convection (IHC), electro-vortex flow (EVF) and swirl flow. Using a steel vessel and a point electrode (Fig. 2) we applied up to $40 \mathrm{~A}\left(0.65 \mathrm{~A} \mathrm{~cm}^{-2}\right)$. We demonstrated that meaningful UDV measurements are possible in a thin fluid layer of only $11 \mathrm{~mm}$ height. For the purpose of comparing experiment and simulation, we developed a model for the ultrasound beam. Further, we derived estimates of the relative importance of thermal convection and electro-vortex flow, and pointed out the limitations of those estimates.

We found the velocity of internally heated convection to be one order of magnitude larger than electro-vortex flow. This relates to our experiment (with the point electrode); it might be different in a real liquid metal battery (LMB). In our experiment, IHC and EVF drive flows of similar shape but in opposing directions. At low current densities, we found thermal convection to dominate the flow structure. The flow became more ordered with increasing current. At higher currents we observed a strong interaction of all flow phenomena. We found a good agreement of simulation and measurements for low currents; as we modeled EVF and IHC separately (and did not account for its interaction), experiment and simulation matched less closely at higher currents. We found the mean velocity of EVF in typical LMBs $(10 \mathrm{~cm}$ side length) to be in order of $\mathrm{mm} / \mathrm{s}$. 
We further observed (azimuthal) swirl flow experimentally at $20 \mathrm{~A}$ for a symmetric experiment, or $10 \mathrm{~A}$ for an asymmetric experiment. The numerical simulations confirmed that vertical magnetic stray fields can cause such flow. However, they did not show a suppression of the (original) poloidal electrovortex flow by the swirling motion. This is not in line with the findings of [63], probably due to the different geometries. These differences deserve further examination. We found the velocity of thermal convection to increase less than linearly with the current. We explained why swirling motion increases approximately linearly and poloidal electro-vortex flow quadratically with current.

The locations and topology of electrical connections affect all four of the flow phenomena we have considered. The Joule heating that drives internally heated convection is a consequence of electrical current, so the placement and size of connections sets the IHC flow. Electro-vortex flow is driven by interactions between the current in the battery and its own magnetic field, both set by the connections with the electric circuit. Swirl flow is driven by interactions between current in the battery and magnetic fields produced by supply lines. And Joule heating of those supply lines can drive RayleighBénard convection.

Battery designers might choose locations and topology of electrical connections to promote mass transfer in the cathodes of liquid metal batteries, thereby allowing faster cycling and preventing formation of intermetallic phases. Rayleigh-Bénard convection may be a suitable phenomenon to use for mixing. External temperature gradients may be imposed using the thermal management system of the cell stack (see [53]). Internally heated convection can produce good mixing, but nearly all Joule heat in a liquid metal battery is produced in the electrolyte, located above the cathode. The resulting stable temperature stratification tends to inhibit internally heated convection, not drive it. Thus IHC may also be problematic for designers. Electrovortex flow seems more promising, because it can produce good mixing and because its magnitude can be adjusted by changing the diameter of the current collector to cause more or less divergence of current. Designers must understand, however, that they make a trade-off: reducing current collector size to promote mixing by electro-vortex flow also increases the resistance of the battery, reducing its voltage and efficiency. Also, real-world electrovortex flows would likely be less vigorous than the ones in our experiments and simulations, which use a narrow current collector resulting in extreme divergence of current. We expect swirl to be less effective at promoting mass 
transfer than the other flow phenomena we have considered because it drives primarily horizontal motion, but vertical mass transfer is most helpful. We also expect swirl to deform the cathode-electrolyte interface substantially in large batteries. For that reason, swirl flow will need special attention as it will surely trigger interface instabilities. Designers should route battery supply wires so as to minimize vertical magnetic fields.

Most importantly, the interaction of internally heated convection and electro-vortex flow deserves further study. In addition, it will be important to reveal how the flow speeds of all phenomena grow with larger currents in larger cells. The appearance and growth of swirl deserves further numerical investigation. In a real LMB cathode, EVF will drive a flow against a stable temperature stratification. This configuration needs to be studied, as well. Finally, flow speed measurements in a real 3-layer LMB would be a great step forward.

\section{Acknowledgments}

This work was supported by the National Science Foundation under award number CBET-1552182, by the Deutsche Forschungsgemeinschaft (DFG, German Research Foundation) under award number 338560565 as well as the Helmholtz-Gemeinschaft Deutscher Forschungszentren (HGF) in frame of the Helmholtz Alliance "Liquid metal technologies" (LIMTECH). The computations were performed on the Bull HPC-Cluster "Taurus" at the Center for Information Services and High Performance Computing (ZIH) at TU Dresden and on the cluster "Hydra" at Helmholtz-Zentrum Dresden-Rossendorf. Fruitful discussions with A. Beltrán, V. Bojarevics, P. Davidson, S. Franke, V. Galindo, G. Horstmann, J. Pal, J. Priede, D. Räbiger, F. Stefani, T. Vogt and T. Wondrak on several aspects of electro-vortex flow and thermal convection are gratefully acknowledged. N. Weber thanks Henrik Schulz for the HPC support.

\section{References}

\section{References}

[1] S. Backhaus, M. Chertkov, Getting a grip on the electrical grid, Physics Today 66 (5) (2013) 42-48. doi:10.1063/PT.3.1979. 
[2] International Energy Agency (Ed.), World Energy Outlook 2013, International Energy Agency, 2013.

[3] H. Kim, D. A. Boysen, J. M. Newhouse, B. L. Spatocco, B. Chung, P. J. Burke, D. J. Bradwell, K. Jiang, A. A. Tomaszowska, K. Wang, W. Wei, L. A. Ortiz, S. A. Barriga, S. M. Poizeau, D. R. Sadoway, Liquid Metal Batteries: Past, Present, and Future, Chemical Reviews 113 (3) (2013) 2075-2099. doi:10.1021/cr300205k.

[4] K. Wang, K. Jiang, B. Chung, T. Ouchi, P. J. Burke, D. A. Boysen, D. J. Bradwell, H. Kim, U. Muecke, D. R. Sadoway, Lithium-antimony-lead liquid metal battery for grid-level energy storage, Nature 514 (7522) (2014) 348-350. doi:10.1038/nature13700.

[5] H. Kim, D. A. Boysen, T. Ouchi, D. R. Sadoway, Calcium - bismuth electrodes for large - scale energy storage, Journal of Power Sources 241 (2013) 239-248.

[6] T. Ouchi, H. Kim, B. L. Spatocco, D. R. Sadoway, Calcium-based multi-element chemistry for grid-scale electrochemical energy storage, Nature Communications 7 (2016) 10999. doi:10.1038/ncomms10999.

[7] S. Poizeau, H. Kim, J. M. Newhouse, B. L. Spatocco, D. R. Sadoway, Determination and modeling of the thermodynamic properties of liquid calcium-antimony alloys, Electrochimica Acta 76 (2012) 8-15. doi: $10.1016 /$ j.electacta.2012.04.139.

[8] T. Ouchi, H. Kim, X. Ning, D. R. Sadoway, Calcium-Antimony Alloys as Electrodes for Liquid Metal Batteries, Journal of The Electrochemical Society 161 (12) (2014) A1898-A1904.

[9] B. Agruss, H. R. Karas, The Thermally Regenerative Liquid Metal Concentration Cell, in: R. F. Gold (Ed.), Regenerative EMF Cells, Vol. 64 of Advances in Chemistry, American Chemical Society, Washington, D.C., 1967, pp. 62-81.

[10] M. S. Foster, S. E. Wood, C. E. Crouthamel, Thermodynamics of Binary Alloys. I. The Lithium-Bismuth System, Inorg. Chem. 3 (10) (1964) $1428-1431$. 
[11] X. Ning, S. Phadke, B. Chung, H. Yin, P. Burke, D. R. Sadoway, Selfhealing Li-Bi liquid metal battery for grid-scale energy storage, Journal of Power Sources 275 (2015) 370-376. doi:10.1016/j.jpowsour. 2014.10.173.

[12] E. J. Cairns, C. E. Crouthamel, A. K. Fischer, M. S. Foster, J. C. Hesson, C. E. Johnson, H. Shimotake, A. D. Tevebaugh, Galvanic Cells with Fused-Salt Electrolytes, ANL-7316, Argonne National Laboratory, 1967.

[13] E. J. Cairns, H. Shimotake, High-Temperature Batteries, Science 164 (1969) 1347-1355.

[14] E. J. Cairns, H. Shimotake, Recent advances in fuel cells and their application to new hybrid systems, Advances in Chemistry 90 (1969) 321-350.

[15] M. S. Foster, C. E. Crouthamel, S. E. Wood, Thermodynamics of Binary Alloys. II. The Lithium-Tin System, J. Phys. Chem. 70 (10) (1966) $3042-3045$.

[16] J. C. Hesson, H. Shimotake, Thermodynamics and thermal efficiencies of thermally regenerative bimetallic and hydride emf cell systems, in: Regenerative EMF Cells, American Chemical Society, 1967, pp. 82104 .

[17] H. Shimotake, G. L. Rogers, E. J. Cairns, Secondary Cells with Lithium Anodes and Immobilized Fused-Salt Electrolytes, Industrial \& Engineering Chemistry Process Design and Development 8 (1) (1969) 5156 .

[18] D. J. Bradwell, H. Kim, A. H. C. Sirk, D. R. Sadoway, Magnesium-Antimony Liquid Metal Battery for Stationary Energy Storage, Journal of the American Chemical Society 134 (4) (2012) 1895-1897. doi:10.1021/ja209759s.

[19] H. Shimotake, E. J. Cairns, Bimetallic galvanic cells with fused-salt electrolytes, in: Advances in Energy Conversion Engineering, ASME, Florida, 1967, pp. 951-962. 
[20] M. S. Foster, G. H. McCloud, E. J. Cairns, Electrochemical studies of the Sodium-Bismuth system, Amer. Chem Soc. (1967) 276 - 281.

[21] L. A. Herédy, M. L. Iverson, G. D. Ulrich, H. L. Recht, Development of a thermally regenerative Sodium-Mercury galvanic system Part I Electrochemical and Chemical Behavior of Sodium-Mercury Galvanic Cells, in: Regenerative EMF Cells, American Chemical Society, 1967, pp. $30-42$.

[22] B. L. Spatocco, P. J. Burke, D. R. Sadoway, Low temperature liquid metal batteries for grid-scaled storage, US patent no. 0099522 A1 (2014).

[23] R. D. Weaver, S. W. Smith, N. L. Willmann, The Sodium-Tin LiquidMetal Cell, Journal of The Electrochemical Society 109 (8) (1962) 653657.

[24] B. Agruss, The Thermally Regenerative Liquid-Metal Cell, Journal of The Electrochemical Society 110 (11) (1963) 1097-1103.

[25] J. Xu, O. S. Kjos, K. S. Osen, A. M. Martinez, O. E. Kongstein, G. M. Haarberg, Na-Zn liquid metal battery, Journal of Power Sources 332 (2016) 274-280. doi:10.1016/j.jpowsour.2016.09.125.

[26] J. Xu, A. M. Martinez, K. S. Osen, O. S. Kjos, O. E. Kongstein, G. M. Haarberg, Electrode Behaviors of Na-Zn Liquid Metal Battery, Journal of The Electrochemical Society 164 (12) (2017) A2335-A2340.

[27] N. E. Holubowitch, S. E. Manek, J. Landon, C. A. Lippert, S. A. Odom, K. Liu, Cathode candidates for zinc-based thermal-electrochemical energy storage: Cathodes for Zn-based thermal-electrochemical energy storage, International Journal of Energy Research 40 (3) (2016) 393399. doi:10.1002/er.3385.

URL http://doi.wiley.com/10.1002/er.3385

[28] N. E. Holubowitch, S. E. Manek, J. Landon, C. A. Lippert, S. A. Odom, K. Liu, Molten Zinc Alloys for Lower Temperature, Lower Cost Liquid Metal Batteries, Advanced Materials Technologies 1 (3) (2016) 1600035. doi:10.1002/admt.201600035. 
[29] D. A. J. Swinkels, Molten Salt Batteries and Fuel Cells, in: J. Braunstein, G. Mamantov, G. P. Smith (Eds.), Advances in Molten Salt Chemistry, Vol. 1, Plenum Press, New York, 1971, pp. 165-223.

[30] M. S. Foster, Laboratory Studies of Intermetallic Cells, in: Regenerative EMF Cells, American Chemical Society, 1967, pp. 136-148.

[31] R. C. Vogel, M. Levenson, E. R. Proud, J. Royal, Chemical engineering division research highlights, Tech. Rep. ANL-7350, Argonne National Laboratory (1967).

[32] F. Stefani, T. Weier, T. Gundrum, G. Gerbeth, How to circumvent the size limitation of liquid metal batteries due to the Tayler instability, Energy Conversion and Management 52 (2011) 2982-2986.

[33] M. Seilmayer, F. Stefani, T. Gundrum, T. Weier, G. Gerbeth, M. Gellert, G. Rüdiger, Experimental Evidence for a Transient Tayler Instability in a Cylindrical Liquid-Metal Column, Phys. Rev. Lett. 108 (244501).

[34] N. Weber, V. Galindo, F. Stefani, T. Weier, T. Wondrak, Numerical simulation of the Tayler instability in liquid metals, New Journal of Physics 15 (043034).

[35] N. Weber, V. Galindo, F. Stefani, T. Weier, Current-driven flow instabilities in large-scale liquid metal batteries, and how to tame them, Journal of Power Sources 265 (2014) 166-173.

[36] W. Herreman, C. Nore, L. Cappanera, J.-L. Guermond, Tayler instability in liquid metal columns and liquid metal batteries, Journal of Fluid Mechanics 771 (2015) 79-114. doi:10.1017/jfm.2015.159.

[37] M. Starace, N. Weber, M. Seilmayer, T. Weier, F. Stefani, S. Eckert, Ultrasound Doppler flow measurements in a liquid metal column under the influence of a strong axial electric current, Magnetohydrodynamics 51 (2015) 249-256.

[38] N. Weber, V. Galindo, F. Stefani, T. Weier, The Tayler instability at low magnetic Prandtl numbers: Between chiral symmetry breaking and helicity oscillations, New Journal of Physics 17 (11) (2015) 113013. doi:10.1088/1367-2630/17/11/113013. 
[39] T. Weier, A. Bund, W. El-Mofid, G. M. Horstmann, C.-C. Lalau, S. Landgraf, M. Nimtz, M. Starace, F. Stefani, N. Weber, Liquid metal batteries - materials selection and fluid dynamics, IOP Conf. Ser.: Mater. Sci. Eng. 228 (012013).

[40] T. Sele, Instabilities of the metal surface in electrolytic alumina reduction cells, Metallurgical and Materials Transactions B 8 (4) (1977) 613-618.

[41] O. Zikanov, Metal pad instabilities in liquid metal batteries, Physical Review E 92 (063021).

[42] N. Weber, P. Beckstein, V. Galindo, W. Herreman, C. Nore, F. Stefani, T. Weier, Metal pad roll instability in liquid metal batteries, Magnetohydrodynamics 53 (1) (2017) 129-140.

[43] N. Weber, P. Beckstein, W. Herreman, G. M. Horstmann, C. Nore, F. Stefani, T. Weier, Sloshing instability and electrolyte layer rupture in liquid metal batteries, Physics of Fluids 29 (5) (2017) 054101. doi: 10.1063/1.4982900.

[44] V. Bojarevics, A. Tucs, MHD of Large Scale Liquid Metal Batteries, in: A. P. Ratvik (Ed.), Light Metals 2017, Springer International Publishing, Cham, 2017, pp. 687-692.

[45] G. M. Horstmann, N. Weber, T. Weier, Coupling and stability of interfacial waves in liquid metal batteries, arXiv:1708.02159.

[46] O. Zikanov, Shallow water modeling of rolling pad instability in liquid metal batteries, arXiv:1706.08589.

[47] Y. Shen, O. Zikanov, Thermal convection in a liquid metal battery, Theoretical and Computational Fluid Dynamics 30 (4) (2016) 275-294. doi:10.1007/s00162-015-0378-1.

[48] T. Köllner, T. Boeck, J. Schumacher, Thermal Rayleigh-Marangoni convection in a three-layer liquid-metal-battery model, Physical Review E 95 (053114). doi:10.1103/PhysRevE.95.053114.

[49] D. H. Kelley, D. R. Sadoway, Mixing in a liquid metal electrode, Physics of Fluids 26 (5) (2014) 057102. doi:10.1063/1.4875815. 
[50] A. Beltrán, MHD natural convection flow in a liquid metal electrode, Applied Thermal Engineering 114 (2016) 1203-1212. doi:10.1016/j. applthermaleng.2016.09.006.

[51] W. Wang, K. Wang, Simulation of thermal properties of the liquid metal batteries, in: Power Electronics Systems and Applications (PESA), 2015 6th International Conference On, IEEE, 2015, pp. 1-11.

[52] D. Bradwell, G. Ceder, L. A. Ortiz, D. R. Sadoway, Liquid electrode battery, US patent no. US 2011/0014505 A1.

[53] D. Bradwell, G. Ceder, L. A. Ortiz, D. R. Sadoway, Liquid metal alloy energy storage device, US patent no. US 9,076,996 B2 (2015).

[54] N. Weber, V. Galindo, J. Priede, F. Stefani, T. Weier, The influence of current collectors on Tayler instability and electro vortex flows in liquid metal batteries, Physics of Fluids 27 (014103).

[55] J. A. Shercliff, Fluid motions due to an electric current source, J. Fluid Mech. 40 (1970) 241-250.

[56] S. Lundquist, On the hydromagnetic viscous flow generated by a diverging electric current, Arkiv fr Fysik 40 (5) (1969) 89-95.

[57] E. V. Shcherbinin, Electrically conducting fluid in self magnetic field of an electric current, Magnetohydrodynamics 11 (1) (1975) 68-74.

[58] C. Sozou, On fluid motions induced by an electric current source, J. Fluid Mech. 46 (1971) 25-32.

[59] V. K. Vlasyuk, Effects of fusible-electrode radius on the electrovortex flow in a cylindrical vessel, Magnitnaya Gidrodinamika 4 (1987) 101106.

[60] V. Bojarevičs, Y. Freibergs, E. I. Shilova, E. V. Shcherbinin, Electrically Induced Vortical Flows, Kluwer Academic Publishers, 1989.

[61] P. A. Davidson, An Introduction to Magnetohydrodynamics, Cambridge texts in applied mathematics, Cambridge University Press, Cambridge ; New York, 2001. 
[62] V. Shatrov, G. Gerbeth, Stability of the electrically induced flow between two hemispherical electrods, Magnetohydrodynamics 48 (3).

[63] P. A. Davidson, D. Kinnear, R. J. Lingwood, D. J. Short, X. He, The role of Ekman pumping and the dominance of swirl in confined flows driven by Lorentz forces, Eur. J. Mech. B 18 (1999) 693-711.

[64] L. A. Volokhonskii, Dynamic boundary layer of electrovortex flow in a cylindrical volume with axisymmetric current supply, Magnetohydrodynamics 27 (4) (1991) 467-470.

[65] V. N. Zhigulev, Some Magneto-Fluid Dynamic Effects in a Finitely Conducting Medium, Reviews of Modern Physics 32 (4) (1960) 828.

[66] V. N. Zhigulev, The phenomenon of ejection by an electrical discharge, Doklady Akademii Nauk SSSR 130 (2) (1960) 280-283.

[67] C. Sozou, H. English, Fluid motion induced by an electric current discharge, Proc. R. Soc. Lond. A. 329 (1972) 71-81.

[68] C. Sozou, Fluid motions induced by an electric current jet, Physics of Fluids 15 (2) (1972) 272. doi:10.1063/1.1693904.

[69] J. P. Narain, Fluid motion caused by conical currents, Physics of Fluids 16 (6) (1973) 940. doi:10.1063/1.1694451.

[70] A. J. M. Jansen, Fluid motions generated by the injection of an electric current, Ph.D. thesis, TU Delft (1986).

[71] A. A. Petrunin, V. N. Shtern, Bifurcation of poloidal field in the flow induced by a radial electric current, Izvestiya Rossiiskoi Akademii Nauk, Mekhanika Zhidkosti i Gaza 2 (1993) 4-11.

[72] H. K. Moffatt, Some problems in the magnetohydrodynamics of liquid metals, Gesellschaft angewandte Mathematik und Mechanik Jahrestagung Goettingen West Germany Zeitschrift Flugwissenschaften 58.

[73] A. Y. Chudnovskii, Modeling electrovortex flows, Magnetohydrodynamics 25 (3) (1989) 337-341.

[74] R. A. Woods, D. R. Milner, Motion in the weld pool in arc welding, Welding Journal 50 (1971) 163-173. 
[75] I. E. Butsenieks, D. E. Peterson, V. I. Sharamkin, E. V. Sherbinin, Magnetohydrodynamic fluid flows in a closed space with a nonuniform electric current, Magnitnaya Gidrodinamika 1 (1976) 92-97.

[76] R. P. Millere, V. I. Sharamkin, E. V. Shcherbinin, Effect of a longitudinal magnetic field on electrically driven rotational flow in a cylindrical vessel, Magnitnaya Gidrodinamika 1 (1980) 81-85.

[77] A. Y. Chudnovskii, Evaluating the intensity of a single class of electrovortex flows MHD, Magnetohydrodynamics 25 (3) (1989) 406-408.

[78] V. V. Boyarevich, V. I. Sharamkin, MHD flows due to current spreading in an axisymmetric layer of finite thickness, Magnitnaya Gidrodinamika 2 (1977) 55-60.

[79] V. G. Zhilin, Y. P. Ivochkin, A. A. Oksman, G. R. Lurin'sh, A. I. Chaikovskii, A. Y. Chudnovskii, E. V. Shcherbinin, An experimental investigation of the velocity field in an axisymmetric electrovortical flow in a cylindrical container, Magnitnaya Gidrodinamika 3 (1986) $110-116$.

[80] D. Räbiger, Y. Zhang, V. Galindo, S. Franke, B. Willers, S. Eckert, The relevance of melt convection to grain refinement in Al-Si alloys solidified under the impact of electric currents, Acta Materialia 79 (2014) 327-338. doi:10.1016/j.actamat.2014.07.037.

[81] Y. H. Zhang, D. Räbiger, S. Eckert, Solidification of pure aluminium affected by a pulsed electrical field and electromagnetic stirring, J. Mater. Sci. 51 (4) (2016) 2153-2159. doi:10.1007/s10853-015-9525-8.

[82] S. Franke, D. Räbiger, V. Galindo, Y. Zhang, S. Eckert, Investigations of electrically driven liquid metal flows using an ultrasound Doppler flow mapping system, Flow Measurement and Instrumentation 48 (2016) 64-73. doi:10.1016/j.flowmeasinst.2015.09.004.

[83] V. Bojarevics, E. V. Shcherbinin, Azimuthal rotation in the axisymmetric meridional flow due to an electric-current source, Journal of Fluid Mechanics 126 (1983) 413. doi:10.1017/S0022112083000245. 
[84] P. Davidson, X. He, A. Lowe, Flow transitions in vacuum arc remelting, Mater. Sci. Technol. 16 (6) (2000) 699-711. doi:10.1179/ 026708300101508306 .

[85] H. Schlichting, K. Gersten, E. Krause, H. Schlichting, H. Schlichting, Grenzschicht-Theorie, Springer, Berlin, 2006, oCLC: 636610285.

[86] V. Shtern, A. Barrero, Bifurcation of swirl in liquid cones, Journal of Fluid Mechanics 300 (1995) 169. doi:10.1017/S002211209500365X.

[87] R. F. Ashour, H. Yin, T. Ouchi, D. H. Kelley, D. R. Sadoway, CommunicationMolten Amide-Hydroxide-Iodide Electrolyte for a LowTemperature Sodium-Based Liquid Metal Battery, Journal of The Electrochemical Society 164 (2) (2017) A535-A537. doi:10.1149/2. $1451702 \mathrm{jes}$.

[88] A. Perez, D. H. Kelley, Ultrasound Velocity Measurement in a Liquid Metal Electrode, Journal of Visualized Experiments 102 (e52622). doi: $10.3791 / 52622$.

[89] I. Kolesnichenko, S. Khripchenko, D. Buchenau, G. Gerbeth, Electrovortex flows in a square layer of liquid metal, Magnetohydrodynamics 41 (2005) 39-51.

[90] H. G. Weller, G. Tabor, H. Jasak, C. Fureby, A tensorial approach to computational continuum mechanics using object-oriented techniques, Comput. Phys. 12 (6) (1998) 620-631.

[91] V. Sobolev, Thermophysical properties of lead and lead-bismuth eutectic, Journal of Nuclear Materials 362 (2-3) (2007) 235-247. doi: 10.1016/j. jnucmat.2007.01.144.

[92] V. Sobolev, Database of Thermophysical Properties of Liquid Metal Coolants for GEN-IV, SCK CEN, 2010.

[93] Nuclear Energy Agency, Handbook on Lead-bismuth Eutectic Alloy and Lead Properties, Materials Compatibility, Thermalhydraulics and Technologies, Tech. Rep. 7268, Nuclear Energy Agency (2015).

[94] A. Oberbeck, Über die Wärmeleitung der Flüssigkeiten bei der Berücksichtigung der Strömungen infolge von Temperaturdifferenzen, Ann. Phys. 7 (271). 
[95] D. D. Gray, A. Giorgini, The validity of the Boussinesq approximation for liquids and gases, Int. J. Heat Mass Transfer 19 (1976) 545-551.

[96] S. Zhang, X. Zhao, General Formulations for Rhie-Chow Interpolation, in: ASME 2004 Heat Transfer/Fluids Engineering Summer Conference, 2004, pp. 567-573.

[97] N. Weber, P. Beckstein, V. Galindo, M. Starace, T. Weier, Electro-vortex flow simulation using coupled meshes, arXiv preprint arXiv:1707.06546.

[98] P. K. Kundu, I. M. Cohen, D. R. Dowling, Fluid Mechanics, Academic Press, 2012.

[99] N. Shi, M. S. Emran, J. Schumacher, Boundary layer structure in turbulent Rayleigh-Bénard convection, J. Fluid Mech. 706 (2012) 5-33. doi:10.1017/jfm.2012.207.

[100] D. Goluskin, Internally heated convection beneath a poor conductor, J. Fluid Mech. 771 (2015) 36-56. doi:10.1017/jfm.2015.140.

[101] O. Kazak, Modeling of Vortex Flows in Direct Current (DC) Electric Arc Furnace with Different Bottom Electrode Positions, Metallurgical and Materials Transactions B 44 (5) (2013) 1243-1250. doi:10.1007/ s11663-013-9899-4.

[102] O. V. Kazak, A. N. Semko, Numerical modeling of electro-vortical flows in a confined volume, J. Eng. Phys. Thermophys. 85 (2012) 1167-1178.

[103] D. R. Atthey, A mathematical model for fluid flow in a weld pool at high currents, Journal of Fluid Mechanics 98 (4) (1980) 787-801.

[104] P. A. Nikrityuk, K. Eckert, R. Grundmann, Y. S. Yang, An Impact of a Low Voltage Steady Electrical Current on the Solidification of a Binary Metal Alloy: A Numerical Study, steel research international 78 (5) (2007) 402-408.

[105] International Atomic Energy Agency, Thermophysical Properties of Materials for Nuclear Engineering: A Tutorial and Collection of Data., International Atomic Energy Agency, Vienna, 2008. 
[106] C. J. Smithells, W. F. Gale, T. C. Totemeier, Smithells Metals Reference Book, 8th Edition, Elsevier Butterworth-Heinemann, Amsterdam ; Boston, 2004.

[107] A. Cramer, J. Pal, G. Gerbeth, Ultrasonic flow measurements in a model of a Czochralski puller, Flow Meas. Instrum. 37 (2014) 99-106. doi:10.1016/j.flowmeasinst.2014.03.008.

[108] J. Pal, A. Cramer, I. Grants, S. Eckert, G. Gerbeth, Physical modelling of temperature fluctuations in a high aspect ratio model of the Czochralski crystal growth, J. Cryst. Growth 432 (2015) 69-77. doi:10.1016/j.jcrysgro.2015.09.009.

[109] J. M. Lopez, F. Marques, Instability of plumes driven by localized heating, Journal of Fluid Mechanics 736 (2013) 616-640. doi:10. 1017/jfm.2013.537.

[110] M. C. Navarro, A. M. Mancho, H. Herrero, Instabilities due to a heating spike, Journal of Physics: Conference Series 64 (2007) 012003. doi: 10.1088/1742-6596/64/1/012003.

[111] M. C. Navarro, A. M. Mancho, H. Herrero, Instabilities in buoyant flows under localized heating, Chaos: An Interdisciplinary Journal of Nonlinear Science 17 (2) (2007) 023105. doi:10.1063/1.2714295.

[112] M. C. Navarro, H. Herrero, Vortices in a cylindrical annulus nonhomogeneously heated: Effect of localized heating on their stability and intensity, Physical Review E 84 (3). doi:10.1103/PhysRevE.84.037301.

[113] M. C. Navarro, H. Herrero, Top-down vortices developed in a cylindrical annulus cooled on the top, Physical Review E 88 (1). doi: 10.1103/PhysRevE.88.015002.

[114] U. Burr, U. Müller, Rayleigh-Bénard convection in liquid metal layers under the influence of a horizontal magnetic field, Journal of Fluid Mechanics 453 (2002) 345-369.

[115] Y. Tasaka, K. Igaki, T. Yanagisawa, T. Vogt, T. Zuerner, S. Eckert, Regular flow reversals in Rayleigh-Bénard convection in a horizontal magnetic field, Phys. Rev. E 93 (4). doi:10.1103/PhysRevE.93. 043109 . 
[116] D. Munger, A. Vincent, A cylindrical model for rotational MHD instabilities in aluminum reduction cells, Theoretical and Computational Fluid Dynamics 22 (5) (2008) 363-382. doi:10.1007/ s00162-008-0082-5.

[117] J. Krautkrämer, H. Krautkrämer, Werkstoffprüfung mit Ultraschall, Springer-Verlag, 1986.

[118] Signal Processing S.A., DOP3000 series User's manual.

\section{Appendix A. Effect of acoustic beam broadening on UDV mea- surements}

In order to precisely compare experimental results gained from UDV measurements to numerical simulations, it is necessary to account for the fact that each ultrasound pulse broadens spatially as it travels away from the emitting transducer, thereby reducing the lateral resolution with increasing distance. Therefore, the measured velocity at each distance interval will be a weighted average over the radial fluid velocity component (pointing toward or away from the transducer) within the width of the beam at that distance interval.

The pressure waves generated by a flat, circular transducer can be thought of as the product of many adjacent point sources in accordance with the Huygens-Fresnel principle. As a result, the ultrasound field in the region near the transducer has a complicated structure. The distance $z$ along the axis of the transducer and the intensity $I_{z}$ follow the relation

$$
I_{z}=I_{0} \sin ^{2}\left(\frac{\pi}{\lambda}\left(\sqrt{r_{T}^{2}+z^{2}}-z\right)\right)
$$

rather than the inverse square law followed by the intensity of spherical waves [117]. Here, $I_{0}$ is the maximum intensity, $\lambda$ is the wavelength, and $r_{T}$ the radius of the transducer. Along the transducer axis, intensity maxima occur when

$$
\frac{\pi}{\lambda}\left(\sqrt{r_{T}^{2}+z^{2}}-z\right)=\left(\frac{1}{2}+n\right) \pi ; n \in \mathbb{N}_{0} .
$$

The axial distances of these maxima are then

$$
z=\frac{r_{T}^{2}-\left(\frac{1}{2}+n\right)^{2} \lambda^{2}}{2\left(\frac{1}{2}+n\right) \lambda} .
$$


The furthest peak of this function occurs when $n=0$ at distance

$$
z_{n f}=\frac{r_{T}^{2}-\left(\frac{1}{4}\right) \lambda^{2}}{\lambda} .
$$

This is the boundary of the near field, or Fresnel zone, beyond which lies the far field, or Fraunhofer zone. In the Fresnel zone, the acoustic field can be approximated as retaining the same cross section as the transducer [118]. In the Fraunhofer zone, the intensity decreases continuously (converging toward the inverse square law at infinity). Moreover, the ultrasound field spreads out conically. This can be quantified by the pressure wave's directivity function $D(\theta)$, which weighs the acoustic intensity with respect to the angle $\theta$ off the transducer axis

$$
D(\theta)=\frac{2 J_{1}\left(k r_{T} \sin \theta\right)}{k r_{T} \sin \theta},
$$

where $J_{1}$ is the Bessel function of the first kind and first order and $k=\frac{2 \pi}{\lambda}$ is the wavenumber [117]. The intensity as a function of the $z$ coordinate and angle is then

$$
\frac{I(z, \theta)}{I_{0}}=\sin ^{2}\left(\frac{\pi}{\lambda}\left(\sqrt{r_{T}^{2}+z^{2}}-z\right)\right)(D(\theta))^{2} .
$$

The angle of divergence is where the intensity's first root lies [117, 118]:

$$
\theta_{0}=\arcsin \left(\frac{0.61 \lambda}{r_{T}}\right)
$$

$I(z, \theta) / I_{0}$ is used as a weighting factor when averaging over the radial velocity components of each point within $\theta_{0}$ at a certain distance from the transducer in the numerical model. This is done to compare it to experimental results. The weighting factor and beam broadening (green planes) are illustrated in Fig. 2. 